\title{
A Remotely Interrogatable Passive Microactuator using SAW Correlation
}

\author{
Ajay C Tikka ${ }^{1}$, Said F Al-Sarawi ${ }^{1}$ and Derek Abbott ${ }^{2}$ \\ ${ }^{1}$ The Centre for High Performance Integrated Technologies and Systems (CHiPTec), \\ ${ }^{2}$ Centre for Biomedical Engineering, \\ The University of Adelaide, SA 5005, Australia. \\ ajay.tikka@adelaide.edu.au, alsarawi@eleceng.adelaide.edu.au,dabbott@eleceng.adelaide.edu.au
}

\begin{abstract}
In this paper, a novel remotely interrogatable ultrasonic microactuator is introduced utilising the complex signal processing functionality of an acoustic wave correlator. Fluid pumping can be achieved at ultrasonic frequencies by electrostatically actuating an edge clamped conducting diaphragm placed above the compressor IDT of the correlator. Secure interrogability of the wireless actuator is demonstrated by encoding the SAW correlator with a $5 \times 2$-bit Barker sequence and quantitatively deducing the code dependent diaphragm motion using finite element analysis. Moreover, the displacement optimization of the microactuator is addressed by the analyses of the device response for various acoustic mode excitations.
\end{abstract}

\section{INTRODUCTION}

Microfluidic pumping and mixing using surface acoustic wave (SAW) devices has been extensively investigated in the past through acoustic streaming [1], [2]. Acoustic streaming based ultrasonic pumps or mixers are less sensitive to the electrical and chemical properties of the fluid when compared to other methods such as electro-osmosis, electrohydrodynamic pumping, magneto-hydrodynamic pumping, and electrophoretic pumping [3]. This is due to the isolation of the microfluidic channel from the electrodes. However, secure actuation of the wireless devices using acoustic streaming is limited only to frequency addressability rather than discrete code addressability, thus rendering them inefficient for remote interrogation applications. This shortcoming is tackled, in this paper, by using a $5 \times 2$-bit Barker sequence encoded SAW correlator and suspending a conducting diaphragm above the compressor interdigital transducer (IDT) of the correlator with an air gap. The correlator response is employed to impart ultrasonic energy on the conducting diaphragm using electrostatic actuation. Thus combining the high frequency operation of the ultrasonic, secure code embedded, acoustic correlator with low power, fast response time, and reliability of electrostatic actuation.

Over the last decade, an increasing number of novel, miniaturized microactuators for biomedical applications have been reported. They have potential applications such as microsurgical tools and drug delivery systems. Several approaches of implantable micropumps have been reported but virtually none of them has reached a mature state yet. Most of these systems are either battery powered or have external pump modules that are connected to the patient by means of a passive port [4]. The use of a battery would place additional constraints on the device size and, furthermore, it would require an electronic circuitry module. Hence, the proposed passive wireless microactuator is highly desirable e.g. for innovative methods in cancer treatment or pain therapy [5]. Moreover, such a delivery system provides a freely programmable, timemodulated release profile to ensure patient safety and comfort in long-term treatment.

Small device dimensions make it typically complicated or even impossible to perform measurements of the physical conditions inside microdevices. To facilitate optimal design of MEMS devices based on such microactuators, efficient simulation schemes for analysing the diaphragm motion are required. The physical behaviour of micro components is best described by partial differential equations, which are typically solved by finite element methods. Furthermore, coupled field simulations are vital for capturing both electro-acoustic and electrostatic-structural interactions in a single finite element run and for taking into account non-linear effects that are inherent in MEMS devices. Hence, in this work, we model the whole structure by employing a direct finite element method (FEM) to verify the functionality of the concept. A systematic approach for modelling and optimising an electrostatic microactuator driven by different code sequences of the interrogating signal and variable acoustic modes of a SAW correlator is presented.

In this paper, the FEM modelling and simulation of a 2dimensional, remotely interrogatable, passive microactuator is presented using a $5 \times 2$-bit Barker sequence encoded SAW correlator. In Section 2, we discuss briefly the principle of operation, the transmitter receiver architecture and the extension of the current design to a micropump structure. Then in Section 3, we present the SAW correlator design, where the dimensions of the structure and materials are discussed. The simulation results comprising the admittance, output voltage response graphs in the frequency domain are outlined in the same section. In section 4, we present the electrostatic model design and the simulation results with diaphragm deflection analysis for variable acoustic modes and different input interrogating code sequences. 


\section{PRINCIPLE OF OPERATION}

The heart of our concept is a SAW correlator, which drives the diaphragm in a secure manner to cause a pulsatile flow of a liquid drug suspended in a carrier fluid. The design of our novel, passive, wireless microfluidic device is motivated by the aim of high-resolution volumetric dosing and the demand for a minimum device size and power consumption.

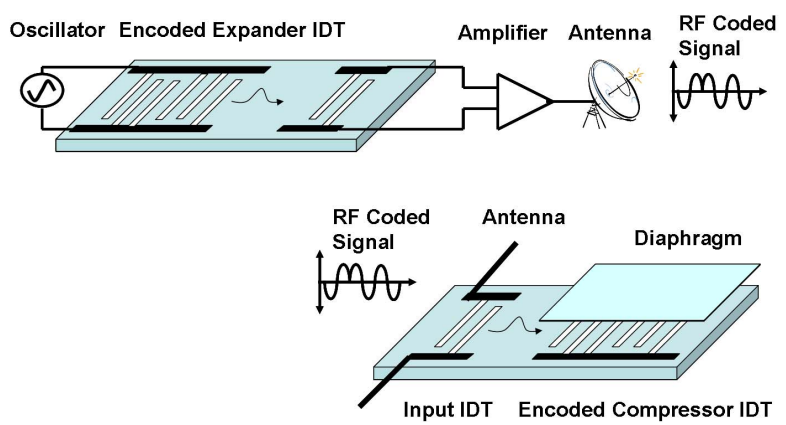

Fig. 1. Wireless microactuator transmitter receiver configuration.

The wireless microactuator concept for which the system has been developed is depicted in Fig. 1. By means of an antenna, the microactuator captures part of the electromagnetic energy to provide power for its own operation. One of the advantages of this approach is the ability to establish bidirectional communication between the microactuator and the interrogator. The power requirement for the communication on the microactuator side is very small, as it is not required to radiate power. Moreover, this concept does not allow simultaneous triggering of several microactuators present in the same electromagnetic field as each device is encoded with a different code. System level protocols, such as those in use in contactless smart-card readers, allows a single controller to communicate with several individually identified microactuators.

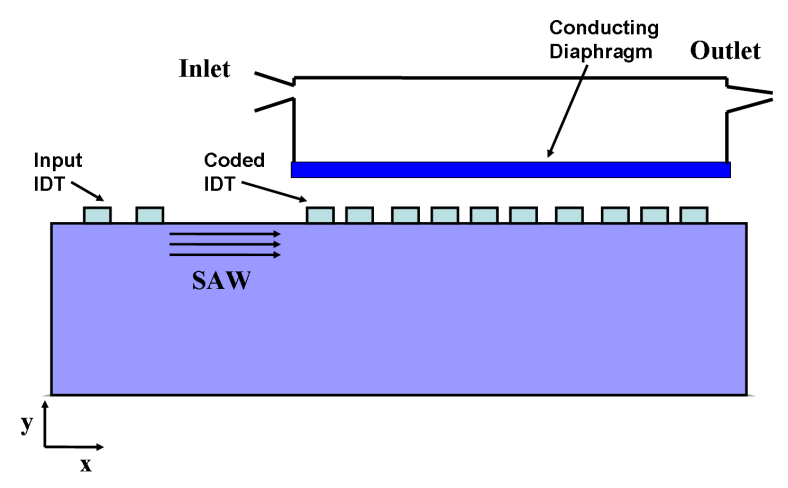

Fig. 2. Microfluidic device in the idle state.

A coded SAW based communication system for microactuation consists of an expander IDT in the transmitter and a compressor IDT in the receiver, as shown in the Fig. 1. A narrow pulse or a sinusoidal waveform is fed to the expander
IDT to generate a coded acoustic signal depending on the geometry of the expander IDT. These acoustic waves propagate through the substrate to the transmitting IDT, which transforms these coded acoustic waves to electrical coded RF signal. The output from the transmitting IDT is fed to an amplifier, to strengthen the signal, and then to a transmitting antenna. The receiver consists of a correlator, the operation of which is explained in the next section, with its input IDT connected to a receiving antenna to intercept the transmitted coded RF signal. The expander in the transmitter is an exact replica of the compressor/coded IDT of the correlator. The coding of the expander and compressor determines the autocorrelation function performed by the correlator. The resultant response of the acoustic device is then electrostatically coupled to a conducting diaphragm, suspended above the compressor IDT with an air gap. Thus the actuation method not only depends on the excitation frequency but also on the code of the transmitted BPSK signal.

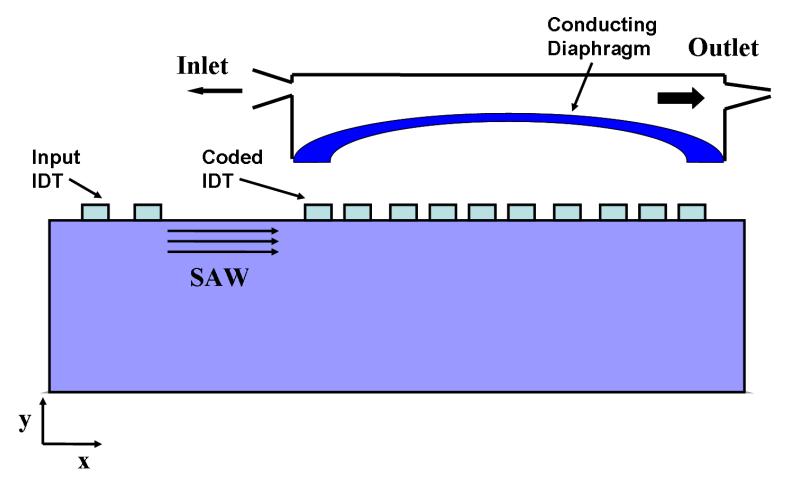

Fig. 3. Microfluidic device in the pumping state.

Diaphragms are often used as a basic building block in micropump and microvalve design. A micropump structure designed using the above described wireless microactuation system is shown in the Fig. 2. In addition to the SAW correlator and the conducting diaphragm the micropump structure consists of a fluid chamber with variable flow resistant inlet and outlet channel. The fluid chamber resembles the one used in a diffuser micropump, researched extensively in the past [6]. In the idle state, when there is no diaphragm deformation, fluid remains stationary in the chamber and there is no outward flow through the outlet channel. On the other hand, in the pumping state, when there is a diaphragm deformation, the fluid is ejected through the outlet with high flow rate as illustrated in Fig. 3. The performance of the micropump such as flow rate, flow direction, and pressure can be flexibly controlled by changing the driving voltage of the interrogating BPSK signal.

The current research primarily focuses on the modeling and optimization of this novel microfluidic structure with emphasis on secure actuation caused by combining electro-acoustic and electrostatic mechanisms. The modelling is carried out using finite element analysis. Both aspects are explained in detail in the subsequent sections. 


\section{SAW CORRELATION}

The need to optimise and verify individual components of the device in order to achieve the desired performance of the integrated assembled system is clearly evident. Hence, we take the approach of modeling and optimizing a SAW correlator first and then proceed to analysing the electrostatic actuation of the diaphragm caused by the correlator.

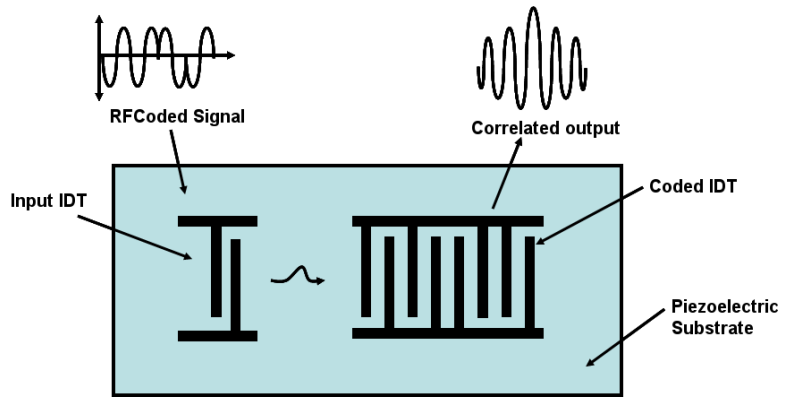

Fig. 4. Surface acoustic wave correlator.

A brief description of the correlator operation is provided here for the sake of clarity. A more detailed description of the same was presented in Ref. [7]. A SAW correlator, as shown in Fig. 4, is a passive pulse compression filter that operates through the correlation of phase shifts in the transmitted RF signal. It consists of an input IDT and a coded output IDT deposited on top of a piezoelectric substrate. The input IDT transduces the coded input RF signal into an acoustic wave. The electrodes of the output IDT are phase coded during construction in such a way that the correlator converts the correctly phase coded acoustic wave to a RF modulated electrical pulse. The device will respond with a correlation peak only when the code of the interrogating RF pulse matches with the embedded code in the output IDT, thus enhancing the processing gain of the correlator by combining the code reception scheme with the high $\mathrm{Q}$ operation of a bandpass filter [8]. For all other transmitted signals with different codes, even the one's excited at the same frequency, the correlator would respond with a pseudo-random noise. The preferred coding scheme is combined Barker sequence, for reasons explained in our previous publication Ref. [9].

\section{A. Geometry}

The modelling of SAW devices is mathematically equivalent to the resolution of the partial differential equations of piezoelectricity for a given excitation. Finite Element Modelling (FEM) is one of the most accurate methods for modelling a SAW device as the complete set of partial differential equations is solved [10]. The modelling of a 2-dimensional, $5 \times 2$-bit Barker sequence encoded, SAW correlator comprising a piezoelectric substrate and two non-uniform input and output IDT's is carried out using ANSYS. Both the input and the output transducers have 20 electrodes each. The input IDT of the structure is modelled as an expander to cause it to generate a coded acoustic wave when a normal pulse or a sinusoidal signal is fed to it. The expander IDT geometry determines the code of the generated acoustic wave. A $5(+++-+) \times 2(++)$ bit Barker sequence is encoded in the output/compressor IDT. This is carried out by coupling the electrodes of the IDT identical to the way they are connected to the positive and negative busbars. The input/expander IDT is an exact replica of the output/compressor IDT when a correlation peak is desired. The response of the device for non-correlating codes can be observed by varying the input IDT finger geometry.

The structure uses a $128^{\circ} \mathrm{YX} \mathrm{LiNbO}_{3}$ piezoelectric substrate of $28 \lambda(\lambda=40 \mu \mathrm{m})$ length, and $5 \lambda$ thickness. Aluminium is assumed for the electrodes with a metallization ratio (MR) of 0.5 , and an electrode thickness of $3 \%$. The material properties of piezoelectric substrate and electrodes are obtained from Ref. [11]. The separation between the IDT's is chosen to be $2 \lambda$. This is found to be an optimal distance to improve the gain and keep the electromagnetic feedthrough, the electromagnetic coupling between the two transducers, under control [12].

\section{B. Simulation Results}

The simulations are aimed at characterising the full scale electromechanical phenomenon of the device, subjected to time and frequency varying excitation. A frequency sweep is performed using harmonic analysis to determine the response of the correlator for different acoustic modes of interest. Apart from the admittance curve and the electric response calculation, analysis of various acoustic modes is carried out with the help of the displacement, electric field and stress contours in $\mu \mathrm{MKS}$ units. In this research, the frequency response of the correlator is obtained by driving the input IDT electrodes with an alternating voltage of $\pm 5 \mathrm{~V}$. The harmonic analysis is carried out for a wide frequency range. Depending on the excitation frequency, various acoustic wave modes such as surface acoustic waves or bulk acoustic waves (BAWs) are excited. They are analysed by observing the contour plots, e.g. displacement, potential, and stress, of the structure.

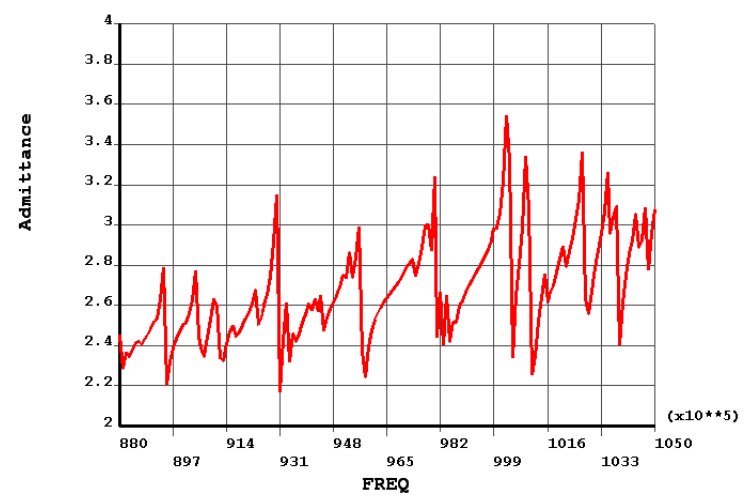

Fig. 5. Admittance magnitude curve.

The complex admittance of the device is computed based on the accumulated charge at the electrodes. As can be seen from the Fig. 5, the magnitude of the complex admittance attains a high value at the resonant frequencies and hence 
exhibits a strong peak. The two admittance peaks to the left of the admittance curve, at frequencies of $89.3 \mathrm{MHz}$ and 90.5 $\mathrm{MHz}$, are the one's corresponding to the symmetric and anti-symmetric surface acoustic wave modes. In the middle part of the admittance curve, bulk acoustic wave modes are observed on the basis of the wave propagation into the bulk of the substrate. An interesting mode, at $100.4 \mathrm{MHz}$, is a leaky surface acoustic wave (LSAW) where both the surface and bulk wave propagations are observed.

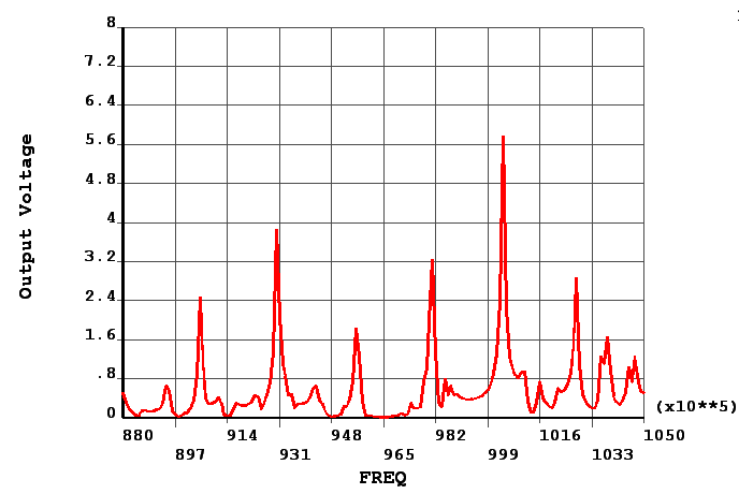

Fig. 6. Magnitude of the voltage across the output IDT.

Fig. 6 shows the output voltage response of the correlator, when the code matches, for different acoustic modes determined by the excitation frequency. The modes definitely impact the response of the correlator and as can be seen from the Fig. 6, the output response is high for modes other than the SAW modes. Hence, it is desirable to operated the correlator at those bulk and LSAW modes to achieve high electromechanical coupling. The response of the correlator is then electrostatically coupled to a conducting diaphragm which is discussed in the next section.

\section{Electrostatic ACtuation}

Electrostatic actuators are prevalent in MEMS devices as they allow large field strengths to be applied at moderate voltages due to the close proximity of the conducting surfaces [13]. Moreover, the fast response times make them an appropriate choice for high speed ultrasonic applications. The structural design and driving parameters of a microactuator should be optimized to meet the required performances with respect to each application. If the targeted application of the microactuator is for a microfluidic device, as in our case, this can be carried out by either changing diffuser channels which shows different flow resistance depending on flow direction or by flexible control of diaphragm displacement and flow rate by changing the applied voltage waveform to the microactuator. The latter approach is taken in this work.

\section{A. Geometry}

In our current model, as shown in the Fig. 7, the actuating field is between a $2 \mu \mathrm{m}$ thick aluminum diaphragm and the output IDT of the correlator, separated by a $2 \mu \mathrm{m}$ air gap.

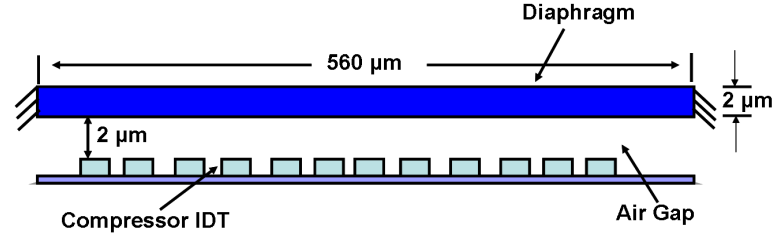

Fig. 7. The electrostatic model.

Aluminum is chosen as the diaphragm material due to its high conductivity and low Young's modulus constant. The length of the edge clamped diaphragm $(560 \mu \mathrm{m})$ is determined by the length of the output IDT of the correlator. Short diaphragm lengths consume less die area and increase the actuator resonant frequency. Longer diaphragms, however, reduce operating voltage requirements [14]. While design rules limit how close a diaphragm can be to a SAW correlator, manufacturability issues limit the thickness of the diaphragm. The flexible electrode or conducting diaphragm is electrically connected to ground, while the voltage at the fixed electrode or output IDT of the SAW correlator is determined by the input interrogating BPSK signal. The electrostatic force that provides this actuation results from the electric field between the interdigitated fingers and the diaphragm. When a voltage of either polarity is applied, deflection of the membrane is possible.

It suffices to carry out a 2-D modelling of the device without compromising on the design accuracy. This is because the movable electrode is assumed to move as a parallel plane, rather than as a flexible membrane. This assumption is reasonable for an actuator having a large width-to-gap ratio as the lateral electrode dimensions are assumed to be much larger than the gap spacing.

\section{B. Simulation Results}

The main objective in the evaluation of the microactuator is to measure the center deflection of the diaphragm when the correlator is interrogated with a matched and mismatched BPSK signal. Fig. 8 shows the center deflection of the diaphragm in the $y$ direction, when the code matches, for different acoustic modes determined by the excitation frequency. Substantial diaphragm displacement can be observed in the vicinity of the excited modal frequencies of the correlator. Hence, the electrostatic analyses of the diaphragm for a widely varying range of frequencies acts as an effective method for displacement optimisation. This allows us to pick the operating mode of interest for displacement optimisation. From Fig. 8, a maximum deflection of the diaphragm of $9.2 \mathrm{~nm}$ can be observed at the LSAW mode at $100.4 \mathrm{MHz}$.

Even though this displacement is less compared to thermal and piezoelectric bimorph actuators the high frequency operation results in high particle velocities [3]. The diaphragm deflection can be further increased by consider a correlator encoded with a longer code, which in turn increasing the diaphragm length. This could not be implemented in the 


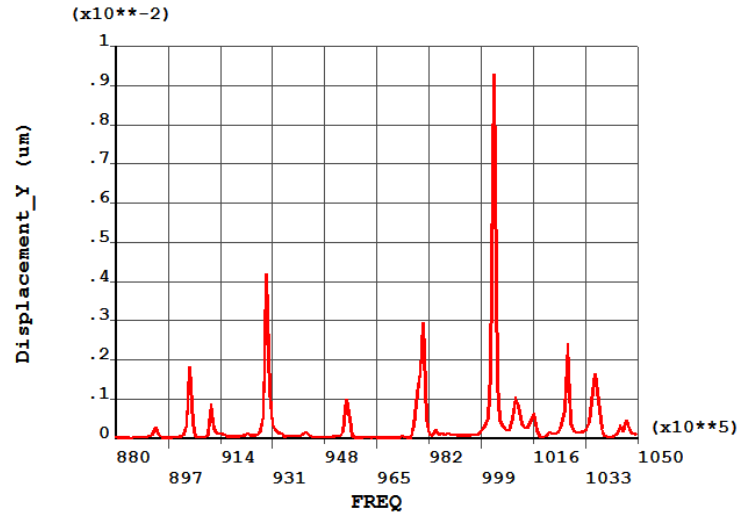

Fig. 8. The center deflection of the diaphragm in the y direction when the code matches.

current work due to the huge constraint imposed by such a correlator modelling technique on the available computational resources.

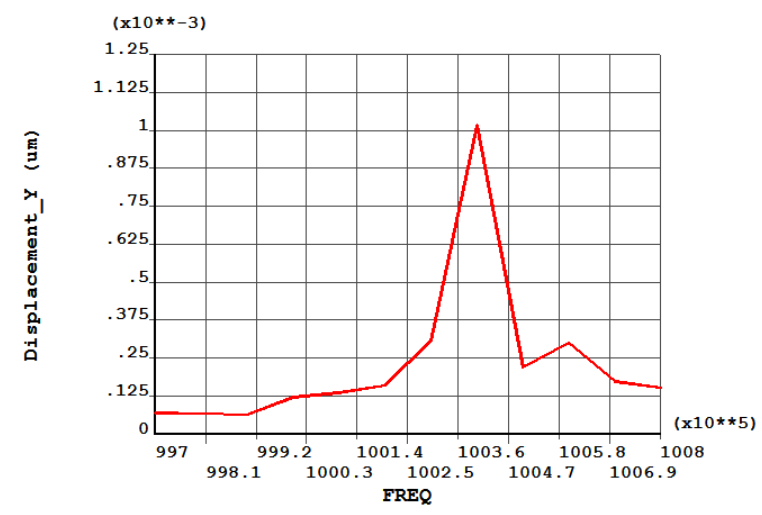

Fig. 9. The center deflection of the diaphragm in the y direction when there is a code mismatches, i.e., for a delay line input.

So far the results are confined to the instance when there is a code match between the input signal, in this model the expander IDT, and the compressor IDT of the correltor. The functionality of the microactuator can only be verified by observing the diaphragm deflection to a mismatched input code. This is carried out by varying the electrode coupling of the expander IDT. The response of the diaphragm to two different codes, a delay line input and other non-correlating input, at the LSAW mode of interest is provided in Figs. 9 and 10. All the other specifications of the model are kept the same except for the mismatched input code.

By comparing these responses with the correlating diaphragm deflection response of the Fig. 8 it can be established that in addition to the excitation frequency, the microactuator's response is determined by the code in the RF signal. Even in the case of a delay line input, when the two codes are very similar, the deflection response of the microactuator is reduced by an order of magnitude 10 to its response to the matched code, as can be seen from Fig. 9.

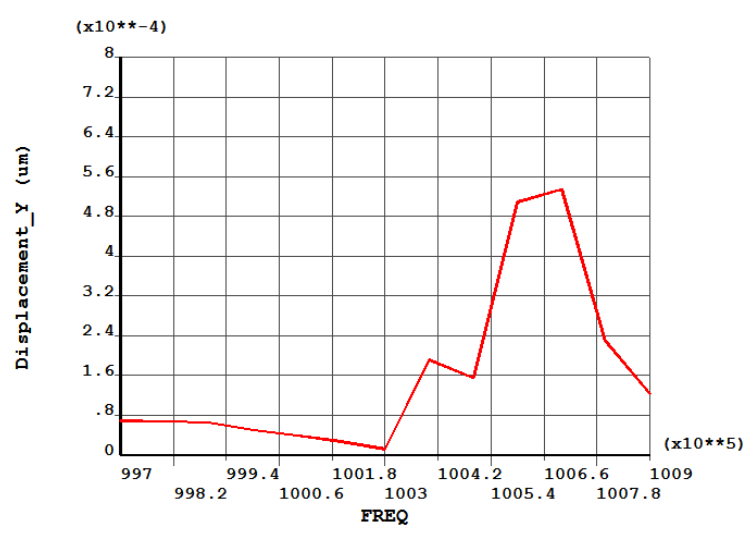

Fig. 10. The center deflection of the diaphragm in the y direction when there is a code mismatches, i.e., for a non-correlating input.

\section{CONCLUSION}

The paper presented the modelling and simulation of a novel wireless passive microactuator by taking into account both the electro-acoustic and electrostatic mechanisms in a single finite element run. The requirements placed by the current and emerging biomedical applications on these devices such as small size and remote interrogability are taken into consideration. The results discussed in this work include: (i) comprehensive FEM modelling of 2-Dimensional, $5 \times 2$ bit Barker sequence encoded SAW correlator, especially the analysis of bulk and LSAW modes, $(i i)$ detailed study of the diaphragm deflection cause by electrostatically coupling the output IDT of the SAW correlator to a conducting diaphragm, actuation optimization by operating the device at the LSAW mode, and analysis of difference in diaphragm deflection when there is a code mismatch. It is concluded that the model appropriately represented the interrogating signal code dependent, and the excited acoustic mode dependent operation of the microactuator and hence enabled the analysis of the diaphragm deflection. In consideration of such actuation analysis by the developed FEM model, a microactuator or a microfluidic device with more optimal performance is expected to be designed for various applications.

\section{ACKNOWLEDGMENT}

The authors would like to thank the Australian Research Council (ARC) and the School of Electrical and Electronics Engineering (University of Adelaide) for the funding and support for the project.

\section{REFERENCES}

[1] H. Yu, J. Kwon, and E. Kim, "Microfluidic mixer and transporter based on pzt self-focusing acoustic transducers," Journal of Microelectromechanical Systems, vol. 15, no. 4, pp. 1015-1024, August 2006.

[2] N. Nguyen, A. Meng, J. Black, and R. White, "Integrated flow sensor for in situ measurement and control of acoustic streaming in flexural plate wave micropumps," Sensors and Actuators. A, vol. 79, no. 2, pp. 115-121, 2000.

[3] V. Kaajakari, A. Sathaye, and A. Lal, "A frequency addressable ultrasonic microfluidic actuator array," in Proc. of 11th International Conference on Solid State Sensors and Actuators Transducers01/Eurosensors XV, June 2001, pp. 958-961. 
[4] L. Cao, S. Mantell, and D. Polla, "Implantable medical drug delivery systems using microelectromechanical systems technology," in Proc. of 1st International Conference on Microtechnologies in Medicine and Biology, October 2000, pp. 487-490.

[5] A. Geipel, A. Doll, F. Goldschmidtböing, B. Müller, P. Jantscheff, N. Esser, U. Massing, and P. Woias, "Design of an implantable active microport system for patient specific drug release," in BioMed'06: Proceedings of the 24th IASTED International Conference on Biomedical Engineering, February 2006, pp. 161-166.

[6] A. Olsson, "Valve-less diffuser micropumps," Ph.D. dissertation, KTH, Signals, Sensors and Systems, 1998

[7] A. Tikka, S. Al-Sarawi, and D. Abbott, "Finite element modelling of SAW correlator," in Proc. of SPIE BioMEMS and Nanotechnology III Conference, vol. 6799, December 2007, p. 679915.

[8] R. Brocato, G. Wouters, E. Heller, J. Blaich, and D. Palmer, "Reconfigurable completely unpowered wireless sensors," in Proc. of 57th Electronic Components and Technology Conference, May 2007, pp. 179183.

[9] A. Tikka, S. Al-Sarawi, D. Abbott, M. Wong, and J. Schutz, "Improving the security and actuation of wireless controlled microvalve," in Proc. of SPIE Smart Structures, Devices, and Systems Conference, vol. 6414, January 2007, p. 64140U.

[10] M. Hofer, N. Finger, G. Kovacs, J. Schölmer, S. Zaglmayr, U. Langer, and R. Lerch, "Finite-element simulation of wave propagation in periodic piezoelectric saw structures," IEEE Trans. on Ultrasonics, Ferroelectrics, and Frequency Control, vol. 53, no. 6, June 2006.

[11] B. Auld, Acoustic Fields and Waves in Solids. Rober E. Krieger, 1990 ( $2^{\text {nd }}$ Edition)

[12] S. Ippolito, K. Kalantar-Zadeh, D. Powell, and W. Wlodarski, "A 3-dimensional finite element approach for simulating acoustic wave propagation in layered SAW devices," in Proc. of IEEE Ultrasonics Symposium, February 2003, pp. 303-306.

[13] W. van der Wijngaart, H. Ask, P. Enoksson, and G. Stemme, "A high-stroke, high-pressure electrostatic actuator for valve applications," Sensors and Actuators. A, vol. 100, no. 2, 2002.

[14] P. Galambos, D. Czaplewski, R. Givler, K. Pohl, D. Luck, G. Benavides, and B. Jokiel, "Drop ejection utilizing sideways actuation of a mems piston," Sensors and Actuators. A, vol. 141, no. 1, pp. 182-191, 2008. 